\title{
Anesthesic Management in Surgery of Congenital Heart Deseases Operated with Cardiopulmonary Bypass at the Pediatric Heart Surgery Center
}

\author{
Fall ML*, Belem PF, Ba EH, Diédhiou M, Leye PA, Diop EHN, Gaye I.
}

UGB $2 S$

DOI: $10.36347 /$ sjams.2021.v09i02.003

| Received: 22.09.2020 | Accepted: 30.09.2020 | Published: 09.02.2021

*Corresponding author: Dr. Fall Mohamed Lamine

Email: tallafal@yahoo.fr

\section{Abstract}

Original Research Article

Congenital heart diseases are quite common. They occur in $0.5-1 \%$ of births. The taking care of children suffering from cardiac malformation must be done early despite the very high operating risks. It requires a suitable center, with adequate medical equipment and special knowledge in both domains anesthesia and surgery. The Pediatric Cardiac Surgery Center in Dakar (CUOMO) is a new public hospital that aims to expand the range of care in the field of cardiology and cardiac surgery to children in Senegal and West Africa; to train the learners in pediatric cardiac surgery for the treatment of simple and complex congenital or rheumatic heart disease. The objective of this study was to evaluate the anesthetic management of congenital heart diseases operated with cardiopulmonary bypass in this center after the first year of activity and to compare the results obtained with data from the literature. We conducted a retrospective, descriptive study over one year from January to December 2018. All patients operated during this period with cardiopulmonary bypass (CPB) for congenital heart disease were included. We collected 80 files of patients operated for congenital heart disease in the center. During the same period, 136 patients had been operated. Surgery for congenital heart disease accounts for $58.8 \%$ of surgical activity. Among these 80 patients, 60 were operated with CPB or a rate of $75 \%$. The average age of our patients was 7.41 years with extremes of 1 and 17 years with a sex ratio of 1.22. A notion of consanguinity was found in 10 of our patients, a frequency of $16.66 \%$. We found 22 cases of cyanogenic heart disease $(36.66 \%)$ and 38 cases of non-cyanogenic heart disease $(63.34 \%)$. The duration of the extracorporeal circulation was on average 82.82 minutes and the average duration of aortic clamping was 58.31 minutes. $70 \%$ of the patients had amines at the end of the procedure. The most common postoperative complication was right heart failure (69\%). The average length of stay in ICU was 4.33 days with extremes of 7 hours to 9 days. We reported the death of one patient carrying mortality at $1.6 \%$. Congenital heart diseases are complex and highly variable in their physiopathology. Due to the improvement of their treatment techniques, their morbidity and mortality have been reduced. However the accessibility of populations to this care is limited by the cost of interventions.

Keywords: Anesthesia, Bypass, Congenital heart disease.

Copyright @ 2021: This is an open-access article distributed under the terms of the Creative Commons Attribution license which permits unrestricted use, distribution, and reproduction in any medium for non-commercial use (NonCommercial, or CC-BY-NC) provided the original author and source are credited.

\section{INTRODUCTION}

Congenital heart disease is quite common. They occur in $0.5-1 \%$ of births [1]. Although countless malformations have been described, three-quarters of them are among the following anomalies: CIV (26\%), persistence of the arterial duct (14\%), CIA (11\%), tetralogy of Fallot (9\%), pulmonary stenosis (8\%), coarctation of the aorta $(6 \%)$, transposition of the large vessels $(6 \%)$ and aortic stenosis $(5 \%)[2]$.

In a quarter of cases, heart disease is associated with one or more other congenital malformations [2]. The management of children with heart defects must be done early despite the very high operating risks. The extent of the residual lesions depends on the type of heart disease but also on the age at which the surgical correction took place: the earlier the child is operated on ( $<3$ years), the less serious the after-effects. For this reason, a total correction is made as much as possible in early childhood and this trend offers children an almost normal quality of life [3].

However, the management of such pathologies imposes on the anesthesiologist a triple constraint:

- Practice anesthesia in a child or a newborn, both of which have anatomical physiological and pharmacological features different from adults.

- Maintain a delicate balance between operations between hemodynamic changes generated by cardiac pathology and compensation mechanisms. 
- Manage post-operative complications generated by extracorporeal circulation.

Anesthesia for pediatric heart surgery has made such progress over the past 25 years, allowing a significant reduction in perioperative mortality.

The objective of this work was to assess the anesthetic management of congenital heart diseases operated under CPB in our department after a first year of activity and to compare the results obtained with data from the literature.

\section{MeTHODS}

We conducted a retrospective study, descriptive over a year going from January to December 2018, in the pediatric heart surgery department CUOMO of Dakar. All patients operated on during this period for congenital heart disease under extracorporeal circulation were included in this study. Patients operated on for rheumatic diseases were excluded from the study, as were congenital heart diseases operated without $\mathrm{CPB}$.

All the patients included in the study had undergone a cardiology consultation with systematically an ECG and a trans-thoracic ultrasound. They also had a cardiac surgery consultation before being sent for the pre-anesthetic visit. After confirmation of fitness for anesthesia, patients were included in the operating program. We considered as risk factors for the surgical intervention the following elements: a complex cardiac lesion, an associated extra cardiac malformation, severe hypoxemia ( $\mathrm{SpO} 2<80 \%)$, too high blood viscosity (ht> $60 \%$ ), PAH (Rap / Ras> 0.5), ventricular failure, young age ( $<3 \mathrm{~kg}$ or $<2$ months), coronary ischemia.

As soon as they arrived in the operating room, patients systematically benefited from monitoring using peripheral oxygen saturation, an electro-cardio scope and non-invasive blood pressure measurement.

In addition to this standard monitoring, patients systematically benefited from the placement of a central venous line and an arterial catheter. They were used respectively to measure central venous pressure, invasive blood pressure and to take iterative samples as needed. A Tran's esophageal ultrasound was systematically performed intraoperatively either by a cardiologist or by the anesthesiologist.

In operation, a blood-saving strategy was systematically carried out by the administration of tranexamic acid. Antibiotic prophylaxis was also performed.

At the end of the intervention, all patients were systematically transferred to intubation for intensive care. They immediately benefited from an immediate post-thoracic ultrasound.

\section{RESULTS}

During the study period, we collected 80 records of patients operated on for congenital heart disease. During this same period 136 patients had been operated on in the department. Congenital heart surgery therefore accounts for $58.8 \%$ of surgical activity.

Among these 80 patients, 60 were operated under CPB, a rate of $75 \%$. For the 20 other patients suffering from congenital heart disease 17 benefited from a PCA treatment and 03 from palliative surgery (two Blalock interventions and one pulmonary artery cerclage).

The average age of our patients was 7.41 years with extremes of 1 and 17 years. The average weight was $21 \mathrm{~kg} \mathrm{+/}-10.8 \mathrm{~kg}$ with extremes of 7 and $60 \mathrm{~kg}$. We counted 27 female and 33 male patients, a sex ratio of 1.22. A concept of inbreeding had been found in 10 of our patients, i.e. a frequency of $16.66 \%$.

We have identified 22 cases of cyanogenic heart disease $(36.66 \%)$ and 38 cases $(63.34 \%)$ of noncyanogenic heart disease. The most common noncyanogenic heart disease was VIC (10 cases) and the most common cyanogenic heart disease was tetralogy of Fallot (19 cases).

On pre-operative clinical examination, no other malformation was associated with heart disease. There were also no predictors of difficult intubation in our patients. Peripheral oxygen saturation in cyanogenic heart disease was on average $81 \%$ with extremes of $61 \%$ and $95 \%$. Two cases of lung infections in progress had been noted during this examination which required treatment

The ECG was performed in all patients. It was abnormal in 46 cases. The anomalies found were: rhythm disturbance type FA, 3 cases of conduction disorder, an enlarged cavity in 37 cases and a conduction disorder associated with an enlarged cavity in 5 cases.

The biological assessment found anemia in 2 preoperative patients. Nine patients had elevated hematocrit and 02 patients had hemostasis associated with elevated hematocrit. These were all patients with cyanogenic heart disease.

None of our patients had preoperative renal function impairment. A risk factor for the intervention was found in 13 patients, a frequency of $21.7 \%$. Of these 13 patients, 12 had cyanogenic heart disease $(92.3 \%)$ and 1 had non-cyanogenic heart disease $(7.7 \%)$.

The existence of some of these risk factors or a lung infection required patient preparation for the procedure. Two patients had received treatment for 
pulmonary hypertension (based on sildenafil) and two others had received treatment for a pulmonary infection (probabilistic antibiotic therapy).

In the operating room, the anesthetic induction performed was inhalation in $61.3 \%$ of cases and intravenous in $38.7 \%$ of cases. For inhalation induction sevoflurane was the halogen used in $100 \%$ of cases. In contrast, intravenous induction showed great variability in the products used alone or in combination. The type of anesthetic interview performed intraoperatively also varied from patient to patient. It was made with variable combinations of morphine in bolus or continuous infusion (fentanyl, sufentanyl), curares, hypnotics (propofol, benzodiazepines, halogenated).

The antibiotic used was cefazolin in 53 cases $(88.33 \%)$ and cefuroxime in 7 cases $(8.83 \%)$. The ACT measurement was variable in the 60 patients operated on CEC. In 23 patients (38.3\%), a measurement was made before the heparin injection, one three minutes after the heparin injection and a final measurement after protamine antagonize. The rest of the patients (37) benefited from a single measurement after heparin injection. The ACT value before the aortic cannulation averaged 494.83 seconds.

Priming for priming the oxygenator and CEC pump circuits was done with crystalloids or colloids in $57 \%$ of the cases. In $43 \%$ of cases, it was associated with packed red blood cells and / or fresh frozen plasma. All patients benefited from blood-cold cardioplegia, the composition of which varied according to the team.

The average temperature during the CEC was $34^{\circ} 5$ Celsius and was measured rectally. The extremes were 24 and 36 degrees. Only one patient was operated on 24-degree deep hypothermia with circulatory arrest to correct a total abnormal pulmonary venous return.

The duration of the CPB was on average 82.82 minutes +/- 33, 41. The extremes were 30 minutes and 197 minutes. The average duration of aortic clamping was 58.31 minutes $+/-24.3$. The extremes were 15 and 125 minutes.

We noted the occurrence of a complication during CEC in 4 patients, a frequency of $6.7 \%$. There was persistent hypotension in one patient and 3 cases of ventricular fibrillation at the time of resumption of cardiac activity, requiring internal electric shock.

Amines were used in 42 patients, or $70 \%$ of the study population (60 patients). 34 patients benefited from a combination of positive inotrope and vasoconstrictor; 6 patients with vasoconstrictors only; two patients' only inotropes.
At the end of the CPB and after stabilization of the hemodynamics, the patients were transferred to intensive care, still intubated with sedation with an average RAMSEY score of 3.45.

A trans-thoracic control ultrasound was performed there as well as biological assessments. When their condition seemed stable, extubating was then considered. The average time for extubating was 12 hours with extremes of 6 hours and 7 days.

In the immediate postoperative period, a complication was found in 36 patients $(60 \%)$. It was about:

- Left heart failure: 3 cases $(8.33 \%)$

- Right heart failure: 25 cases $(69.44 \%)$

- Pulmonary arterial hypertension: 4 cases (11.11\%)

- Arrhythmias: 1 case $(2.77 \%)$

- Hemostasis disorders: 19 cases (52.77\%), including thrombocytopenia in 5 cases, low prothrombin level in 9 cases and a combination of the two in 5 cases (The total exceeds 36 because the same complications can be found in the same patient).

The average duration of use of amines in resuscitation was 3 days with extremes of 2 and 5 days. The evolution in resuscitation was interspersed with complications in resuscitation in 3 patients $(5 \%)$.

The first patient had been operated on for pulmonary stenosis. At postoperative day 2, he presented severe right heart failure with a right ventricle-pulmonary artery gradient at $147 \mathrm{mmhg}$. He was re-intubated and received treatment with milrinone. In view of the improvement in symptoms, he was extubed on day 5 and transferred to hospital on day 13 .

The second patient had been operated on for a $\mathrm{CAV}$. The evolution in intensive care was marked in the first hours by severe hypoxia. The bedside ultrasound showed that the inferior vena cava drains into the left atrium responsible for hypoxia. The surgical resumption was made at H3. The evolution was favorable and the patient was transferred to day 4 in hospital.

The last patient was operated on for a tetralogy of Fallot. In the hours following the operation, she suffered significant bleeding leading to shock, then cardiac arrest and death at $\mathrm{H} 7$.

The mortality during surgery for congenital heart disease under CEC was therefore $1.60 \%$. The average length of stay in intensive care was 4.33 days with extremes of 7 hours to 9 days. 


\section{DISCUSSION}

In our study, we collected 80 files of patients operated on for congenital heart disease over a period of one year. Only 60 of them were operated under CPB. The number of patients operated on during this period was 136 . In a study looking at congenital heart disease surgery in adults Haddad [4] in 2015 had found a total of 540 patients over a period of 16 years. This makes a frequency of about 33 patients per year. This difference can be explained by the fact that congenital heart disease surgery is more frequent and more indicated in childhood. Also very few patients not operated on in childhood reach adulthood or have fairly advanced forms which then become inoperable. Only a part can still benefit from surgery at this age. In 2011 FALL in a retrospective study looking at anesthetic management and resuscitation of congenital heart diseases operated by a Swiss team in Dakar had found 19 patients over a period of 6 months [5].

The average age of patients operated on in our department was 7.41 years with extremes of 1 and 17 years. These data are always superior to those recommended in the literature (surgery before 3 years).This would limit the risk of sequelae and improve the quality of life [3]. The main difficulty we encounter in the pursuit of this objective is twofold; First, the diagnosis of heart disease in our context is most often made at an already advanced age, but also the care both anesthetically and the requirements of the CPB of young children $(<2$ months or $3 \mathrm{~kg})$ constitute a major obstacle. However, we note a clear progression in the management of these pathologies in Dakar because FALL in 2011 had found an average age of 9.6 years with extremes of 6 and 17 years [5].

We counted $52.5 \%$ of female patients and $47.5 \%$ of male patients for all congenital heart diseases operated on (with or without CPB) in the department during the study period. These figures are roughly equal to those found in a Belgian study by Moons in 2002 [6]. They found $51 \%$ girls and $49 \%$ boys. Mbaye [7] in a study done in Dakar on congenital heart disease in adults also found a female predominance (64\%).

In our study, we found a notion of inbreeding in $16.66 \%$ of children with congenital heart disease. Inbreeding is a recognized source of genetic defect. This reinforces the hypothesis that one of the causes of congenital heart disease is genetic. Fall ML had found a frequency of inbreeding of around $15.78 \%$ [5]. These results are close to those we found.

The most frequent congenital heart diseases in our study were the tetralogy of Fallot $31.66 \%$; the CIA $16.66 \%$ and the CIV $16.66 \%$. In other studies [8, 9] different prevalence were found. A classification was made of the most frequent congenital heart diseases for 100 diagnosed cases. Then came the CIV 26\%, the CIA $12 \%$ and the ductus arteriosus $8 \%$. The tetralogy of
Fallot which was the most frequent in our study there was a prevalence rate of only $8 \%$.

The preoperative clinical examination found no malformation associated with heart disease. However in the literature it is described that in a quarter of the cases, the heart disease is associated with one or more other congenital malformations [10].

The EKG was normal in 14 patients. The most common abnormality was an isolated enlarged cavity in 37 patients, a frequency of $46.25 \%$ (atrial or ventricular hypertrophy). There was also an isolated conduction disorder or associated with an enlarged cavity in 8 patients (right or left branch block or fascicular block). A rhythm disorder was found in only one patient. It was a complete arrhythmia by atrial fibrillation. In contrast, in the study by Mbaye [11] the most common abnormality was rhythm disturbance at a frequency of $18 \%$.

The biological assessment showed a disturbance in 13 patients. There was moderate anemia in 2 patients.

Eleven patients had an elevated hematocrit level greater than $60 \%$. These patients all had cyanogenic heart disease. This polycythemia is due to the stimulation of erythropoietin secretion by chronic hypoxia [12]. It significantly increases the viscosity of the blood and often involves the presence of associated coagulation disorders [12]. Thus, among these 11 patients, we found 2 cases of blood hemostatic disorders associated with elevated hematocrit.

In addition, the search for electrolyte disturbances and in particular potassium is recommended in children receiving digitalis, diuretic treatment and by converting enzyme inhibitor [12]. In our study we did not find any case of ionic disorder or disruption of renal function.

In our study we found the existence of at least one risk factor in 13 patients, a frequency of $21.7 \%$. Of these 13 patients, 12 had cyanogenic heart disease and 1 had non-cyanogenic heart disease (CIV + HTAP). These figures reflect the difficulty of managing cyanogenic heart disease compared to non-cyanogenic.

The existence of some of these risk factors or of a lung infection required patient preparation for the procedure. The presence of a recent airway infection can not only lead to bronchial hyper responsiveness, but also a change in pulmonary vascular resistance which can then be poorly tolerated in children with congenital heart disease [12]. Thus 2 patients had benefited before the intervention from treatment for a pulmonary infection by antibiotic therapy. Two other patients had $\mathrm{PAH}$ and received treatment with sildenafil to lower lung pressures before surgery. 
Twenty to thirty percent of children with congenital heart disease develop heart failure during their clinical course [12]. In our study, we did not find any cases of cardiac decompensation preoperatively. This is explained by the fact that patients even before being scheduled for surgery are followed by a cardiology team. She was in charge of the therapeutic adjustment before referring the patients once their condition seemed stable.

The induction performed was inhalator in $61.3 \%$ of cases and intravenous in $38.7 \%$ of cases. For inhalation induction sevoflurane was the halogen used in $100 \%$ of cases. In contrast, intravenous induction showed great variability in the products used alone or in combination. This is explained by the fact that the anesthesia teams varied a lot. Induction and maintenance were done not only according to the habits of each team but also according to the patient's pathology and clinical condition. Indeed there is no simple recipe applicable to all children in anesthesia for congenital heart disease; the extreme variability of pathologies means that several techniques are possible, based on the use of a main agent and auxiliary agents. The choice is guided by cardiac pathology, age, duration of CPB, degree of hypothermia and postoperative predictions [13].

In our study, the patients who underwent extracorporal circulation surgery (60 patients) all benefited from the administration of tranexamic acid intraoperatively. The technique for delivering bolus or continuous tranexamic acid also varied from team to team. Junejo F. et al. in a study comparing bolus and continuous infusion of tranexamic acid to reduce bleeding in children undergoing cyanogenic congenital heart disease surgery concluded that there was no statistically and clinically significant difference between the 2 techniques [14]. The parameters they studied were the amount of postoperative bleeding and the time the thoracotomy was closed.

All operated patients had received cefazolinbased antibiotic prophylaxis in $90 \%$ of the cases and cefuroxime in $10 \%$ of the cases. Cefazolin is the antibiotic recommended in heart surgery [15]. Cefuroxime was used due to the lack of availability of cefazoline. We noted only one case of post-operative infection $(1.25 \%)$. It was an infectious.

Our patients benefited from an injection of 300 IU / $\mathrm{kg}$ of heparin and an ACT measurement before the aortic cannulation. The recommended ACT time is approximately 400 seconds [16]. In our study, the average delay for ACT was 494.83 seconds. Which corresponds to the data in the literature. However, some teams carried out a systematic measurement of the ACT before the heparin injection and another at the end of the CEC after protamine anatomization. This practice reassures you about the neutralization of all the heparin administered to the patient but also eliminates this cause in case of postoperative bleeding. However, it remains limited by the availability of ACT tubes.

The volume of the CEC (priming or priming) is 2 to 4 times greater than the circulating volume of the child. The extreme dilution imposed by the large priming volume decreases oncotic pressure and hematocrit, which increases tissue edema and lowers peripheral arterial resistance. It decreases the concentration of platelets and coagulation factors [10]. To reduce this hem dilution it was associated in $43 \%$ of the cases with packed red blood cells and / or fresh frozen plasma in the priming of our patients. This addition was mainly made in children less than 5 years of age in whom the hem dilution is greater.

All of our patients have had blood cold cardioplegia. Evidence of superiority of cardioplegia in blood compared to cardioplegia in crystalloids has long been the subject of controversy. However, cardioplegia in the blood gradually imposed itself so that it is no longer discussed especially in risky situations (HVG, reduced ventricular function). However, the mechanisms of the benefit of blood cardioplegia on the crystalloid vectors remain incompletely elucidated. Note, however, that hem dilution and hypothermia combine to limit the amount of oxygen supplied to the myocardium. In addition, the heart consumes oxygen in real time and we can therefore hope for the creation of an aerobic environment only during the few contemporary minutes of the cardioplegia infusion. It is therefore likely that the protective effects of blood cardioplegia are based on the rheological effects of blood and the cardio protective effects of some of its constituents (proteins, buffer systems, antioxidants) [10].

The temperature was on average $34^{\circ} 5$ Celsius and measured at the rectal level. The extremes were 24 and 36 degrees. Only one patient was operated on 24degree deep hypothermia with circulatory arrest for correction of RVPAT. The drop in cellular metabolism in the cold provides a certain degree of protection of the organs and makes it possible to extend the time of ischemia by a variable duration depending on the temperature [10]. In the study by Fall ML et al. the average temperature during $\mathrm{CEC}$ was $35^{\circ} \mathrm{C}$ with extremes of 34 to $36^{\circ} \mathrm{C}$ [5].

In our study the duration of the CEC was on average 82.82 minutes $+/-33,41$. The extremes were 30 minutes and 197 minutes. The average duration of aortic clamping was 58.31 minutes $+/-24.3$. The extremes were 15 and 125 minutes. There is no maximum duration of $\mathrm{CPB}$, but it is recognized that the frequency of complications increases over time [16]. Fall ML had found similar values with an average aortic clamping time of $50 \mathrm{~min}$ and an average duration of CEC of $90 \min [5]$. 
During CEC, the complications encountered in our study were: one case of persistent hypotension requiring vasoconstrictor injections and three cases of ventricular fibrillation when resuming cardiac activity requiring internal cardiac shocks.

During cardiac surgery, the use of vasopressors and inotropic agents for loading is dictated by observation of the heart in the operating field and at TEE, by analysis of central arterial and venous pressures, and by the hemodynamic modifications imposed at the end of CEC [10]. In our study, amines were used in 42 patients, ie $70 \%$ of the patients: 34 patients benefited from a combination of positive inotrope and vasoconstrictor; 6 patients with vasoconstrictors only; 2 patients' only inotropes. In the study by Fall ML et al. 53\% of the patients had benefited from amines [5].

At the end of CEC and after stabilization of the hemodynamics, the patients were transferred to intensive care, still intubated and sedated. There was a trans thoracic control ultrasound as well as biological assessments. When their condition seemed stable, extubating was then considered. The average time for extubating was 12 hours with extremes of 6 hours and 7 days. According to some authors, rapid extubating $(<4$ hours postoperative) avoids complications linked to mechanical ventilation (barotrauma, respiratory infections, obstruction of the endotracheal tube); it allows early mobilization and shortens the length of stay in intensive care [10].

Although pleasant, this theory puts the patient at risk of hypoventilation or respiratory arrest. In short the duration of the postoperative mechanical ventilation and the time for extubating are still debated, extubating on the table or in intensive care a few hours later each having their followers $[17,18]$.

The most common complications in immediate post-SCC in our study were right heart failure $(69.44 \%)$ hemostasis disorders $(52.77 \%)$ and PAH $(11.11 \%)$. These observations are consistent with the data in the literature which define right dysfunction as the dominant complication [10].

The short and medium term evolution was interspersed with complications in intensive care in 3 patients (5\%). Among these 3 patients, 2 had a favorable evolution. The last patient died, giving a death rate of $1.6 \%$. Haddad A. et al. [4] in their study on congenital heart disease in adulthood found mortality slightly above $2.4 \%$. Diouf E. [19] and Beye SA. [20] in studies also carried out in Dakar in patients operated on for congenital heart disease by foreign teams found mortalities of $19 \%$ and $14 \%$ respectively. Our results attest to the progress made in the management of congenital heart disease, especially since the patients were mainly operated by the local team.
The average length of stay in intensive care was 4.33 days with extremes of 7 hours to 9 days. Fall ML found an average length of stay in intensive care of 6 days [5]. This shows a marked improvement in the post-operative management of these types of pathologies.

\section{CONCLUSION}

Congenital heart diseases are complex and very variable in their pathophysiology. With the improvement of techniques and knowledge their morbidity and mortality has greatly decreased. To improve the care of children suffering from these pathologies, consultation between the anesthesiologist, the pediatric cardiologist and the surgeon is essential. The choice of an anesthetic technique for a child with a heart disease will be based on a good knowledge of the malformation and its repercussions, cardiovascular and pulmonary effects and possible interactions of the agents at our disposal. Good follow-up in intensive care in the postoperative remains essential for a good evolution. Antenatal diagnosis of heart defects would improve this management, but it is still insufficient today in our regions.

\section{Current State of Knowledge}

- Congenital heart diseases are fairly frequent pathologies in our countries.

- Their surgical management is limited due to the lack of suitable infrastructure, of personnel specialized in this field both anesthetically, surgically and cardio logically.

- Contribution of our study to knowledge

- It allows us to report on the results of our first year of activity in the first structure in West Africa allowing surgery for congenital heart diseases.

- The mortality from these pathologies can be reduced if care is taken early, with a team specialized in this area and suitable equipment.

\section{Conflicts of interest}

The authors do not declare any conflict of interest.

\section{REFERENCES}

1. Brickner EM, Hillis LD, Lange RA. Congenital heart disease in adults. First of two parts. N Engl J Med. 2000; 342: 1-12

2. Daniels SR. Epidemiology. In: LONG WA. Fetal and neonatal cardiology. Philidelphia: WB Saunders.1990, 430

3. Castaneda AR, Jonas RA, Mayer JE, Hanley F. Cardiac surgery for newborns and infants. Philidelphia: WB Saunders. 1994, 23

4. Haddad A. Surgery for congenital heart disease in adulthood. About 540 cases. Ann Cardiol Angeiol (Paris), http://dx.doi.org/10.1016/j.ancard.2015.02.005 
5. Fall ML, Leye PA, Ba PA, Bah MD, Ndiaye PI, Ciss AG. Perioperative management of congenital heart diseases in Senegal. Rev Afr Anesth Méd Urg. 2012; 17(3): 3-10

6. Moons P, Sluysmans T, De Wolf D, Massin M, Suys B, Benatar A. Congenital heart disease out of 111,225 births in Belgium: prevalence of births, treatment and survival in the 21 st century. Acta Paediatr. 2009; 98: 472-7.

7. Mc Auliffe JJ. Storage of the myocardium. In: LAKE CL, ed. Pediatric cardiac anesthesia, 3rd edition. Stamford: Appleton and Lange. 1998, 25983

8. Baumgartner H, Bonhoeffer P, De Groot NMS, de Haan F, Deanfield JE, Galie N. ESC guidelines for the management of congenital heart disease in adults (new version 2010). Eur Heart J. 2010; 31: 2915-257.

9. Warnes C, Williams R, Bashore T, Child J, Connolly H. ACC / AHA 2008 Guidelines for the Management of Adults with Congenital Heart Disease. J Am Coll Cardiol. 2008; 52: e143-263.

10. Chassot P. Summary of cardiac anesthesia [online]. Available at:

$<$ http://www.precisdanesthesiecardiaque.ch/index.h tml> (accessed 03/23/2018).

11. Mbaye A, Bodian M, AA. Ngaïdé. Congenital adolescent and adult heart disease: care in a general cardiology department in Senegal. Annals of cardiology and angiology. 66 (2017) 217-222
12. Habre W. Assessment of anesthesia in children with congenital heart disease. Archives of paediatrics. 2006, 13(6): 754-757

13. Chassot PG. Anesthesia in cardiac surgery. In: Ecoffey C, Hamza J, Meistelman C. Pediatric anesthesiology. Paris: Flammarion. 1997 : 183-202

14. Junejo F, Akhtar MI, Hamid M. Comparison between intravenous bolus and tranexamic acid (TXA) infusion to reduce bleeding in pediatric surgeries of congenital cyanotic heart disease (CHD). J Coll Physicians Surg Pak. 2018 Mar; 28 (3): 180-183

15. Amara Y, Baujard C. Antibiotic prophylaxis for heart surgery. In: Resuscitation anesthesia protocols. Le kremlin - Bicêtre: Mapar editions. 2013: 272

16. Bonnet F, Soulier A., Spielvogel C. Anesthesia in cardiac surgery. In: The book on internal anesthesiology. Flammarion editions. Paris: Flammarion Medecine- sciences. 1998: 395-407.

17. Dinardo JA. Disadvantages: extubation in the operating room after pediatric heart surgery. J Cardiothorac Vasc Anesth. 2011; 25: 877-9

18. Mittnacht AJC. Pro: early extubation after surgery for congenital heart disease. J Cardiothorac Vasc Anesth. 2011; 25: 874-6

19. Diouf E. Anesthesiologist in charge of congenital heart disease in Dakar. Assessment of 21 cases 1994-1996 Rev Afr Med Urg. 1997: 2.1: 1-8

20. Beye SA. Anesthesia - resuscitation in the complete healing of congenital heart diseases Afr. Ann. Thorac. Cardiovasc. Surg. 2010; 5(1): 29-33. 